\title{
O que se diz sobre os MOOCs? A produção científica brasileira sobre os Massive Open On-line Courses nos últimos 10 anos
}

\author{
Eliane Almeida do Carmo ${ }^{1}$, Carolina Lopes Araújo², Gardênia Silva Abbad³, Pedro Paulo \\ Murce Menezes ${ }^{4}$
}

eliane.adm@gmail.com, carolinaaraujo@unb.br, gardenia@unb.br, pemeneses@yahoo.com.br

${ }^{1}$ Fundação Osvaldo Cruz (FIOCRUZ - Brasília), Avenida L3 Norte, s/n, Campus Universitário Darcy Ribeiro, Gleba A, 70904-130 , Brasília - DF, Brasil

${ }^{2}$ Faculdade UnB Planaltina, Universidade de Brasília, Vila NS de Fátima - Vila Nossa Sra. de Fátima, 73345010, Brasília - DF, Brasil

${ }^{3}$ Instituto de Psicologia, Campus Universitário Darcy Ribeiro ICC Sul, 70910-900, Brasília - DF, Brasil

${ }^{4}$ Faculdade de Economia, Administração e Contabilidade - Universidade de Brasília - Campus Darcy Ribeiro

- Prédio da FACE Asa Norte - CEP: 70910-900, Brasília - DF, Brasil

DOI: 10.17013/risti.33. 1-15

Resumo: O incremento do acesso à internet e a ampliação do acesso à tecnologia de informação e comunicação (TICs) ajudaram a criar um contexto propício ao desenvolvimento de Massive Open On-line Courses (MOOCs). Os MOOCs, constituem uma ferramenta acessível de educação mediada por computadores que favorece a democratização e a ampliação do alcance do ensino à distância. Este estudo visou desenhar um panorama das produções científicas brasileiras sobre MOOCs nos últimos 10 anos. Apoiada em um levantamento bibliométrico seguido de revisão sistemática da literatura, as análises revelaram pequena produção sobre MOOCs no Brasil e que os pesquisadores não são autores assíduos na temática. Foi observado que são poucos os grupos de pesquisas brasileiros sobre o tema, mas são comuns parcerias interinstitucionais nesses estudos. Observou-se que a investigação brasileira sobre MOOCs é recente, pouco consolidada, mas apresenta demanda por novas pesquisas.

Palavras-chave: Revisão sistemática de literatura; MOOCs; WebQDA; produção científica brasileira; educação à distância.

MOOCs, what do we talk about? Brazilian scientific production on the Massive Open On-line Courses in the last 10 years

Abstract: The improvement in Internet access and wider access to information and communication technology (ICTs) created favorable context for Massive Open Online Courses (MOOCs). MOOCs are considered an accessible and inexpensive education tool able which contributes to democratization of educational processes through computer-mediated learning process. This work draws a panorama of the 
of Brazilian scientific production on the subject by presenting a bibliometric study joint to a systematic literature review of the studies published in Brazilian journals in the last 10 years. The analyzes revealed that there are few studies on MOOCs in Brazil and that the researchers do not publish frequently about this subject. There are few Brazilian research groups on MOOCs, but that interinstitutional partnerships are common. Finally, the Brazilian research on MOOCs is recent, lacks consolidation but demands new studies about MOOCs.

Keywords: Literature systematic review; MOOCs; webQDA; Brazilian scientific production; distance education.

\section{Introdução}

O processo de ensino/aprendizagem vive um complexo momento de profunda transformação propiciada pelo amplo acesso à internet e pelo desenvolvimento de tecnologias de informação e comunicação (TICs) (Luís, Rocha, \& Marcelino, 2017, p. 55). Segundo Ruano, Congote e Torres (2016, p. 16), o uso generalizado de computadores pessoais, os telefones celulares e os tablets reconfigurou as maneiras de se receber, interpretar, administrar e transmitir a informação. Nesse contexto surgem novas tecnologias de educação à distância para a aprendizagem aberta - open learning - que se caracterizam pelo amplo acesso aos recursos pedagógicos via internet e pela construção de conhecimento por meio de interações sociais e educacionais. Novas oportunidades formativas ganharam forma nos ambientes virtuais, tais como os Massive Open On-line Couses (MOOCs), simulações de realidade virtual, treinamento assíncrono, jogos em rede, além de aprendizagem informal e auto-estudo.

Os MOOCs surgiram há cerca de uma década caracterizados por sua flexibilidade; livre acesso (abertos); baixo (ou nenhum) custo para os usuários e potencial de alcance massivo (Barin \& Bastos, 2013). Eles se realizam em Ambientes Virtuais de Aprendizagem (AVAs), por meio de ferramentas da web e/ou por redes sociais que apresentam como proposta a integração das tecnologias em rede. Martins, Leite e Ramos (2017) explicam que os MOOCs surgem inicialmente como uma proposta científica e acadêmica de educação à distância articulada à uma proposta econômica de internacionalização da educação.

Os MOOCs ainda não possuem um padrão de funcionamento definido e seu futuro acadêmico é incerto (Cid Bastos \& Biagiotti, 2014). Fontana e Leffa (2018) afirmaram que as plataformas MOOC têm avançado inexoravelmente em um intervalo de tempo relativamente curto, e a tendência para os próximos anos é que se amplie ainda mais. Os MOOCs assumem relevância especial na atual conjuntura de conhecimento amplamente disponível e grande competitividade (Araujo et al., 2017; Brites \& Rocha, 2017; Dal Forno \& Knoll, 2013; Fassbinder, Delmaro, \& Barbosa, 2014; Gomes \& Barbosa, 2016; Mejías, Gema, \& Melo, 2013; Oliveira, 2017; Teixeira, Mota, Morgado, \& Spilker, 2015).

Os MOOCs têm se mostrado ferramentas importantes para viabilizar o acesso a conteúdos de renomadas universidades (Cid Bastos \& Biagiotti, 2014; Zhang, Peck, Hristova, Jablokow, Hoffman, Park, \& Bayeck, 2016), que, em muitos casos, estariam fora de alcance de ampla parcela de potenciais estudantes na modalidade presencial. 
Ao propiciarem o acesso a todos os estudantes aos mesmos cursos, por meio do acesso à internet, os MOOCs são apresentados como uma proposta para democratização do ensino (Cid Bastos \& Biagiotti, 2014; Nascimento, Cardoso, \& Rocchietti, 2013).

A compreensão acerca dos MOOCs pode fornecer importantes informações sobre desterritorialização da educação (Dal Forno \& Knoll, 2013). Porém, apesar dos prometidos benefícios dos MOOCs para estreitar o fosso digital e promover a equidade nas oportunidades educacionais, o uso de MOOCs ainda se mostra muito aquém das possibilidades tecnológicas disponíveis (Ma \& Lee, 2018) e pouco se conhece sobre sua eficiência nos processos de formação-educação (Noe, Clarke, \& Klein, 2014), especialmente no contexto dos países em desenvolvimento.

O presente trabalho objetiva traçar o panorama dos estudos sobre MOOCs realizados no Brasil, desde o surgimento dos MOOCs em 2008 até 2018. Para tanto, foram considerados os estudos publicados no período em periódicos das áreas de Administração, Educação, Psicologia e Saúde Coletiva, por serem essas as áreas mais correlatas ao fenômeno estudado, bem como sendo as áreas de atuação, interesse e proficiência científica dos autores deste trabalho. São objetivos secundários deste trabalho apresentar a institucionalização da pesquisa no Brasil, descrevendo grupos de pesquisa, autores e instituições envolvidos no tema, além de apontar as lacunas de pesquisa sobre o tema.

É importante ressaltar que este trabalho se deriva de um estudo de maior escopo, ainda inédito, sobre impacto de aprendizagem em ambientes virtuais abertos. Uma versão resumida deste trabalho foi publicada nas Atas do $8^{\circ}$ Congresso Ibero-Americano em Investigação Qualitativa - CIAIQ 2019 (do Carmo, Araújo, Murce, \& Abbad, 2019) e o presente artigo rediscute as análises, mas de modo que não altera substancialmente os resultados analíticos apresentados no trabalho anterior - visto se tratar da mesma base de dados. Entretanto, este artigo amplia a descrição dos métodos de investigação utilizados e detalha os procedimentos de tratamento e questionamento aos dados auxiliados pelo software webQDA.

Este artigo se estrutura por uma seção de revisão teórica na qual são apresentados os principais conceitos e o histórico do surgimento dos MOOCs; seguida por uma seção que apresenta os métodos, protocolos e procedimentos aplicados neste, os quais fundamentam as análises e discussões apresentadas na seção subsequente; e finaliza-se por uma seção de considerações finais na qual são apresentadas as lacunas na investigação sobre os MOOCs e na qual se propõe uma agenda de pesquisa futura sobre o tema.

\section{Histórico e principais conceitos sobre MOOCs}

Três fases distintas marcam o desenvolvimento da EaD: primeiramente tem-se os estudos por correspondência; depois, vieram as mídias eletrônicas aplicadas a processos formativos à distância e mais recentemente surgiram os cursos massivos e abertos online - os MOOCs (Gomes \& Barbosa, 2016). O primeiro MOOC foi oferecido por George Siemens, Stephen Downes e Dave Cormier na Universidade de Manitoba, no Canadá, em 2008 (Souza \& Cypriano, 2016; Teixeira et al., 2015). O curso, intitulado "Connectivism 
and connective knowledge" foi oferecido a 25 alunos em regime presencial e 2.300 alunos on-line (Souza \& Cypriano, 2016). Desde então, os MOOCs cresceram consideravelmente e continuam crescendo em popularidade (Rodrigues, Ramos, Silva, \& Gomes, 2016).

Os modelos de MOOCs mais recorrentes são os x-MOOCs, que mais se assemelham às tradicionais aulas presenciais nos quais a figura do professor ainda exerce o papel central (Barin \& Bastos, 2013) e os c-MOOCs, nos quais os próprios alunos geram a maioria das atividades, escrevendo suas próprias reflexões em blogs e plataformas sociais e compartilhando com seus pares.

Enquanto os c-MOOCs são de natureza conectivista e adotam o conceito de "aberto" tal como definido no campo da educação aberta onde todos colaboram e constroem o conhecimento, os x-MOOCs entendem "aberto" como um sinônimo de "gratuito" (Teixeira et al., 2015). Não há consenso nos estudos sobre MOOCs quanto à massividade desses cursos. Vela (2016) ressalta que os MOOCs não são massivos no sentido de quantidade de participantes, mas que assim se adjetivam quando se considera que os participantes provêm de múltiplas instituições e diversos países e diferentes níveis de formação. Sabe-se, entretanto, que "a acessibilidade dos ambientes virtuais de aprendizagem, para pessoas com diferentes perfis e necessidades, assume uma preocupação relevante”, uma vez que contribuem para a utilização mais ampla e abrangente dos espaços virtuais de aprendizagem (Luís et al., 2017, pp. 59 -60).

No que se refere ao acesso aberto, Alevizou (2015), Rodrigues, Ramos, Silva e Gomes (2016) e Rambe e Moeti (2017) ressaltam que os MOOCs são vistos como promotores de objetivos sociais por meio da ampliação do acesso à educação. Mas esses autores também consideram que imperam motivações tecno-econômicas nas decisões quanto à oferta dos MOOCS. Grande parte dos MOOCs se fundamentam em conhecimentos de base ocidental, servindo para a exportação da uma epistemologia científica hegemônica. Isso tem estimulado críticas de que os MOOCs podem favorecer uma forma sutil de neocolonialismo intelectual (Martins, Leite \& Ramos, 2017; Rambe \& Moeti, 2017). Outras críticas aos MOOCs denunciam a perversidade do viés mercadológico que define a oferta desses cursos, o que se faz evidente quando alguns cursos gratuitos são usados para publicidade de outros serviços - on-line ou não pagos (Fontana \& Leffa, 2018).

Além disso, pouco se sabe sobre a efetividade da experiência de aprendizagem de alunos com diferentes habilidades, motivações e disposições, e são escassas as inovações com ganho significativo no aprendizado em relação aos métodos tradicionais (Rodrigues et al., 2016). Frequentes críticas pautam a pouca interação e o feedback pobre entre professores e alunos e entre os próprios estudantes (Fontana \& Leffa, 2018). A maioria dos MOOCs não são desenhados para favorecer experiências de aprendizagem colaborativas (Teixeira et al., 2015) e apresentam altas taxas de evasão (Higashi, Schunn, \& Flot, 2017).

Grandes incertezas pairam sobre o futuro dos MOOCs (Cid Bastos \& Biagiotti, 2014). É inegável que eles criam oportunidades para atividades mais flexíveis e interativas e para novas estratégias de avaliação (Barin \& Bastos, 2013). 


\section{Métodos de investigação}

Esta revisão sistemática foi guiada pelo Protocolo de Cronin, Ryan e Coughlan (2008) e seus procedimentos (passo-a-passo) estão sintetizados no Quadro 1:

\begin{tabular}{lll}
\hline $1^{o}$ passo & Formular a pergunta de pesquisa & $\begin{array}{l}\text { "O que tem sido publicado sobre MOOCs no Brasil } \\
\text { nos últimos 10 anos e por quem?” }\end{array}$ \\
\hline $2^{o}$ passo & $\begin{array}{l}\text { Definir os critérios para a coleta } \\
\text { de dados (inclusão dos periódicos } \\
\text { consultados) }\end{array}$ & $\begin{array}{l}\text { Periódicos com classificação Qualis Capes igual ou } \\
\text { superior a B2 em Psicologia, Saúde, Administração e } \\
\text { Educação. }\end{array}$ \\
\hline $3^{o}$ passo & $\begin{array}{l}\text { Selecionar e acessar os itens que } \\
\text { compõem o corpus de pesquisa }\end{array}$ & $\begin{array}{l}\text { Artigos indexados com as palavras-chave: Educação } \\
\text { aberta; MOOC; MOOCs; ou Massive Open Online } \\
\text { Courses. }\end{array}$ \\
\hline $4^{o}$ passo & $\begin{array}{l}\text { Analisar o corpus de pesquisa e } \\
\text { sintetizar os achados }\end{array}$ & $\begin{array}{l}\text { Gerar categorias de classificação dos artigos e } \\
\text { classificá-los com a ajuda do software WebQDA de } \\
\text { análise qualitativa. }\end{array}$ \\
\hline $5^{o}$ passo & Disseminar os resultados da pesquisa & Redigir e publicar o presente artigo \\
\hline
\end{tabular}

Quadro 1 - Revisão sistemática de literatura pelo Protocolo de Cronin, Ryan e Coughlan (2008)

O corpus de pesquisa deste trabalho foi composto em três etapas: primeiramente uma busca exploratória inicial no Google Acadêmico permitiu identificar as palavras-chave que posteriormente embasaram as buscas nos periódicos brasileiros pelos artigos que seriam analisados. Nessa primeira etapa, foram considerados 30 trabalhos dos quais foram extraídas 87 palavras-chaves. Dessas palavras, 51\% não se repetiam. As palavraschave mais frequentes foram: MOOC (11 vezes); Educação aberta (5); MOOCs (4); e Massive Open Online Courses (3). A segunda etapa se deu pela seleção dos periódicos dos quais seriam extraídos os artigos que comporiam o corpus de pesquisa, ou seja, todos os periódicos indexados pelo sistema Qualis Capes (evento de classificação Quadriênio 2013-2016 - o mais recente no momento da pesquisa) com nota igual ou superior a B2 nas áreas de Administração, Educação, Psicologia e Saúde (considerou-se a maior classificação, quando classificados em mais de uma área). A terceira etapa foi a coleta dos artigos com as palavras-chave selecionadas na etapa anterior. As buscas incluíram as publicações de janeiro de 2008 a novembro de 2018, ou seja, desde o surgimento dos MOOCs até a finalização da coleta de dados para este estudo. Buscou-se artigos que contivessem nos títulos, resumos ou palavras-chave os termos: "MOOC", "MOOCs", "Massive Open Online Course" ou "Educação aberta". Foram encontrados 149 artigos, dos quais 113 foram excluídos por não terem relação direta com o tema. Esses trabalhos tratavam de EAD ou tecnologias de informação e comunicação de uma forma geral, com menção superficial aos MOOCs.

Após essa seleção, restaram, então, 36 artigos, os quais foram submetidos à leitura rigorosa. Então, foram eliminados outros sete artigos por não tratarem especificamente do tema. O corpus de pesquisa definitivo se compôs por 29 artigos estritamente relacionados ao estudo dos MOOCs. O Quadro 2 sintetiza os números de artigos que compõem o corpus de pesquisa deste estudo, separados por área de conhecimento de classificação do periódico em que o trabalho foi publicado. 


\begin{tabular}{llllll}
\hline Área & $\begin{array}{l}\text { Trabalhos } \\
\text { selecionados } \\
\text { (1a busca) }\end{array}$ & $\begin{array}{l}\text { Exclusões } \\
\text { (título / } \\
\text { resumo }\end{array}$ & $\begin{array}{l}\text { Exclusões } \\
\text { (leitura } \\
\text { do texto) }\end{array}$ & $\begin{array}{l}\text { Composição } \\
\text { final do } \\
\text { corpus }\end{array}$ & Observações \\
\hline $\begin{array}{l}\text { Educação } \\
108\end{array}$ & 83 & 3 & 22 & $\begin{array}{l}\text { 5 experiências } \\
\text { internacionais / traduções }\end{array}$ \\
\hline Administração & 14 & 5 & 4 & 5 & $\begin{array}{l}\text { 2 experiências } \\
\text { internacionais }\end{array}$ \\
\hline Psicologia & 25 & 24 & 0 & 1 & 1 artigo de tradução \\
\hline Saúde Coletiva & 2 & 0 & 1 & 1 & $\begin{array}{l}\text { 1 em inglês, em parceria } \\
\text { brasileira }\end{array}$ \\
\hline Total & 149 & 113 & 7 & 29 & \\
\hline
\end{tabular}

Quadro 2 - Síntese quantitativa da busca de trabalhos sobre MOOCs em periódicos brasileiros com classificação Qualis Capes maior ou igual a B2

$\mathrm{Na}$ revisão bibliométrica dos 29 artigos selecionados, utilizou-se das seguintes categorias de análise: 1) Periódico de publicação do artigo; 2) Área do Periódico; 3) Ano de publicação do artigo; 4) Autores; 5) Quantidade de autores; 6) Tipo de artigo (teórico ou teórico-empírico); 7) Natureza da Pesquisa (descritiva, explicativa ou exploratória); 8) Palavras-chave; 9) Principais resultados; e 10) Lacunas reveladas pelo estudo.

Com relação aos autores, buscou-se as informações relacionadas às 4.1) instituições de vinculação na data da publicação do artigo; 4.2) país da instituição de vinculação do autor; 4.3) unidade federativa (UF) da instituição de vinculação do autor (se instituição brasileira); 4.4) titulação máxima do autor; e 4.5) quantidade de trabalhos na temática ou em temática relacionada. As informações sobre os autores, quando não disponíveis nos artigos, foram buscadas no currículo lattes, para o caso dos brasileiros e estrangeiros que estavam cadastrados na plataforma. Para os pesquisadores estrangeiros que não estavam cadastrados no lattes, a busca foi realizada pelo nome completo dos autores e a consulta de seus trabalhos no Google Acadêmico. Buscou-se também informações sobre 4.4) grupos de pesquisa cadastrados no Diretório dos Grupos de Pesquisa no Brasil voltados para MOOCs e aos quais os autores poderiam estar vinculados a tais grupos.

Os artigos classificados como teóricos-empíricos (22 no total, pois sete artigos derivaram-se de investigação puramente teórica), também foram categorizados segundo: 7.1) Abordagem de pesquisa (qualitativa, quantitativa e mista); 7.2) Origem dos dados (primário, secundário ou misto); 7.3) Delineamento (experimento, quaseexperimento, correlacional ou sem delineamento); 7.4) Recorte temporal (transversal ou longitudinal); 7.5) Método (survey/levantamento de opiniões, quase-experimento, experimento, estudo de caso, observação participante, pesquisa documental e pesquisaação); 7.6) Amostragem (censitária, probabilística ou não-probabilística e rígida ou flexível); 7.7) Perfil amostral (alunos, professores, gestores, executivos, plataformas); 7.8) Instrumentos de procedimento de coleta de dados (questionário, roteiro de entrevista, roteiro de grupo focal, roteiro de observação, roteiro de análise documental e roteiro de análise de fóruns); 7.9) procedimentos de coleta (online, presencial, por telefone); e 7.10) técnicas de análises de dados (estatística descritiva, estatística inferencial, análise de conteúdo e análise de discurso). 
Após analisado o panorama bibliométrico das publicações, foi realizada uma análise qualitativa interpretativa dos objetivos e conteúdo dos artigos, a fim de mapear a evolução temática dos estudos sobre MOOCs publicados nos periódicos brasileiros que compõem o corpus desta pesquisa. Para essa etapa de análise qualitativa, foi usado o software webQDA de análise qualitativa. Já a análise bibliométrica foi majoritariamente realizada com o auxílio do software MS Office Excel.

\subsection{Usando o webQDA para tratamento e análise dos dados}

Os 29 artigos que compuseram o corpus de pesquisa deste trabalho foram importados para o webQDA como fontes internas em formato PDF e separadas em pastas segundo a área de classificação do periódico de que foram extraídas. Foram, então, criadas classificações das fontes, que funcionam como um "rótulo descritivo para todo o documento" (Souza, Costa, Moreira, Souza, \& Freitas, 2016) e não apenas para parte dele (como funcionariam os códigos e os descritores). As classificações permitem filtrar as fontes a partir de seus atributos. A Figura 1 apresenta os itens de classificação dos artigos selecionados, que se apoiam nas seguintes categorias: 1) Periódico de publicação do artigo; 2) Área do Periódico; 3) Ano de publicação do artigo; 5) Quantidade de autores; 6) Tipo de artigo (teórico ou teórico-empírico); 7) Natureza da Pesquisa (descritiva, explicativa ou exploratória).

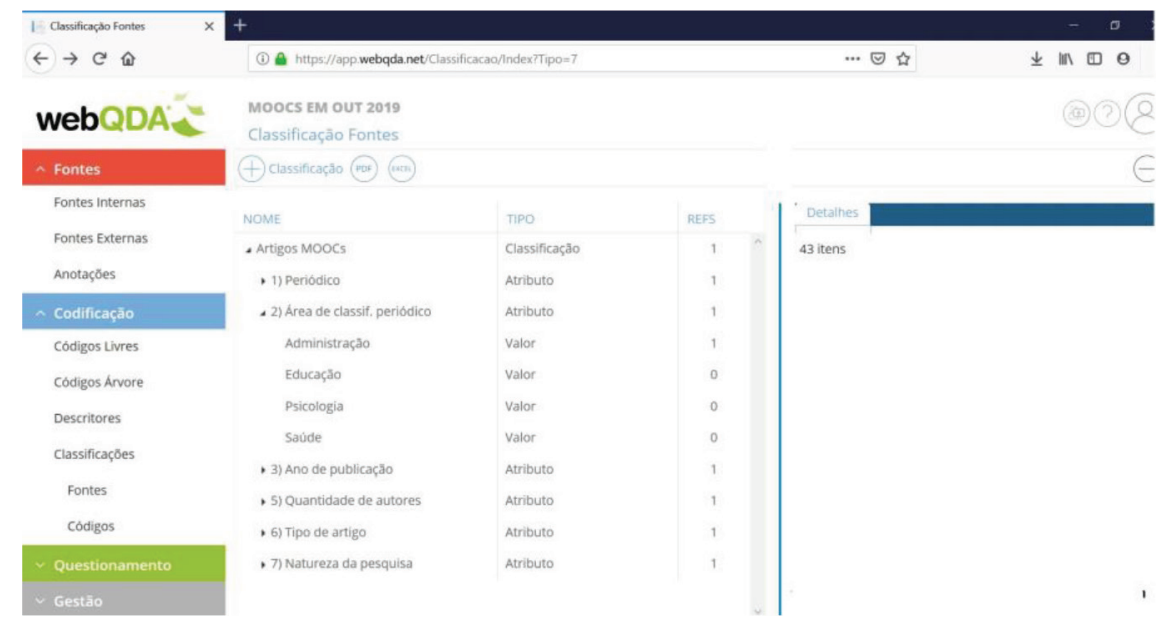

Figura 1 - Classificação por atributos de artigos científicos na interface do software webQDA

Após terem sido criada as categorias de classificação e definidos seus respectivos valores de atributos, fica fácil aplicá-las sobre as fontes. Basta, para tanto, acessar a pasta das fontes e, no canto direito da tela, na aba realçada na Figura 2 com a seta vermelha, atribuir os valores dos atributos de classificação criados anteriormente. Vale lembrar que é importante gravar a operação pressionando o botão com o símbolo do disquete. 
O leitor deve ter notado que, dentre as classificações de fontes, não foram incluídas as categorias 5) Autores; 8) Palavras-chave; 9) Principais resultados; e 10) Lacunas. Tal exclusão é devida à natureza dos dados dessas categorias, que presumem grande variedade de valores e, portanto, têm pouca utilidade para a filtragem das fontes. Um recurso do webQDA útil para destacar e identificar nos artigos esses tipos de dados é a geração de códigos. Diferentemente das classificações (que se aplicam a toda a fonte), códigos podem ser aplicados aos trechos das fontes que distinguem as informações relevantes para a categorização.

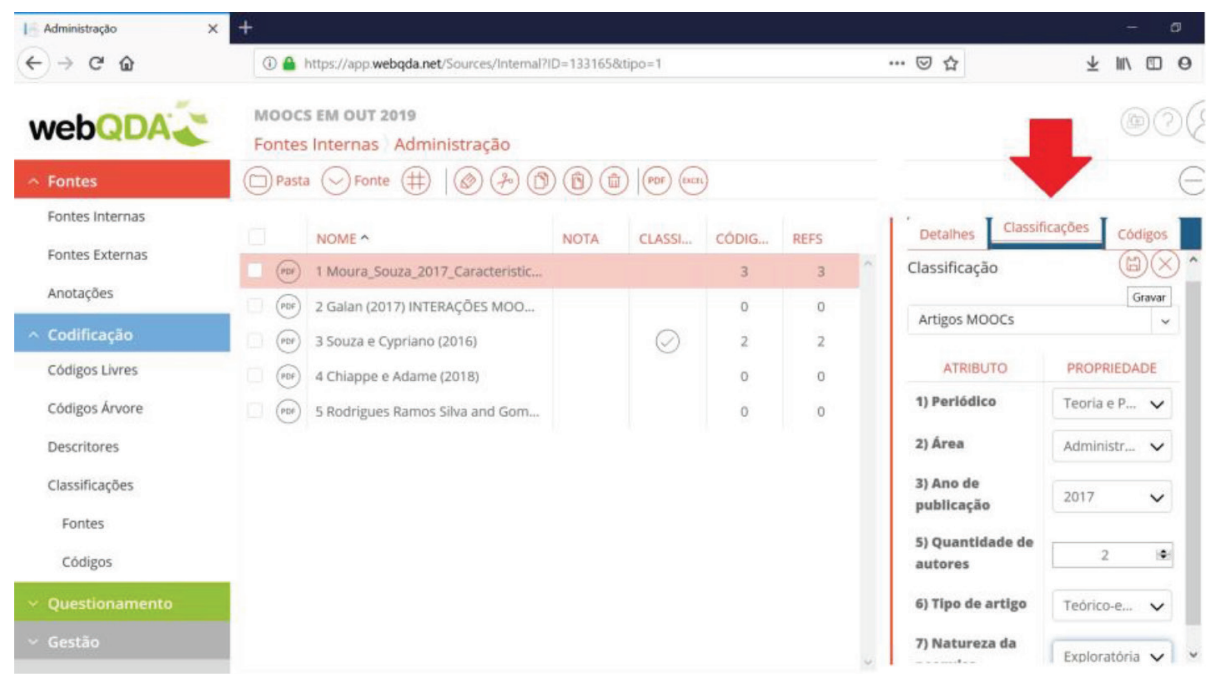

Figura 2 - Atribuir classificação de fonte na interface do software webQDA

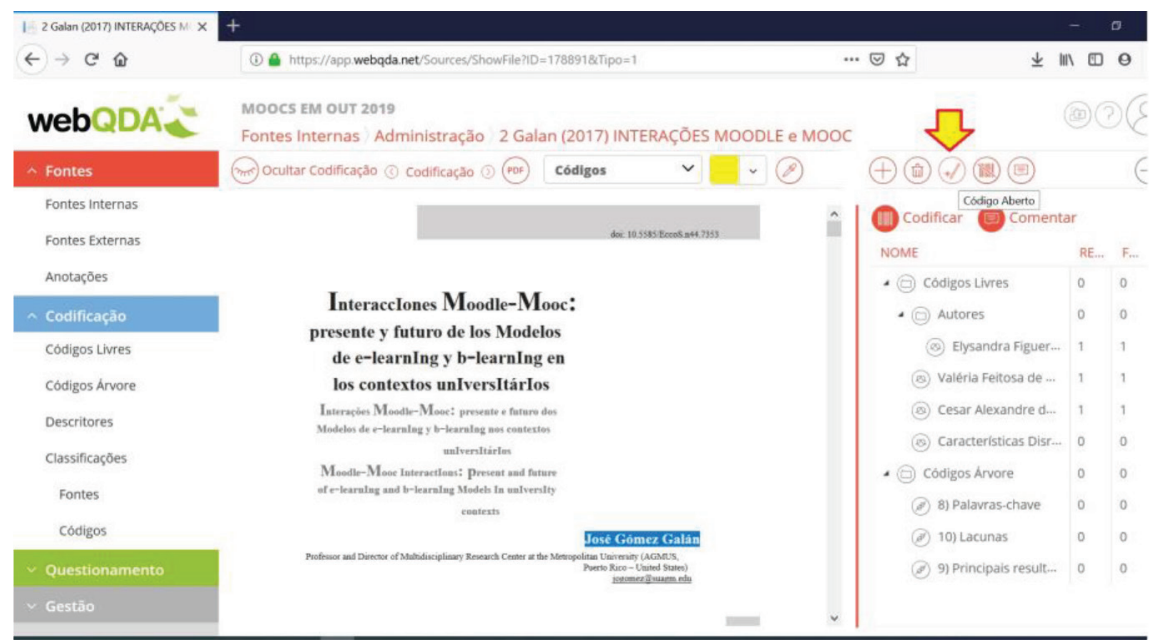

Figura 3 - Criar código livre a partir da marcação do texto da fonte 
Para a codificação dos autores dos artigos analisados, foram geradas categorias na medida em que se avançou na leitura dos textos. A ferramenta "códigos abertos" do webQDA que permite gerar novos códigos livres a partir da marcação direta no texto dos artigos importados no formato PDF. Assim foi possível nomear as categorias pelos nomes dos autores. Essa ferramenta agilizou enormemente o processo de codificação em códigos livres e evita erros de grafia ao se extrair o nome das categorias diretamente do texto original. A ferramenta para a geração de “códigos abertos” está realçada na Figura 3 pela seta amarela.

O processo de geração de códigos livres com o nome dos autores pelo uso da ferramenta "código aberto" do webQDA permitiu identificar 89 autores no total dos artigos em análise. Sobre os códigos livres relativo aos autores foram aplicadas "classificações de códigos" por dois atributos: titulação do autor - com seis valores possíveis: doutorado; doutorando (em curso); mestrado; mestrando (em curso); graduação e sem informação - e a instituição de origem do autor - que neste estudo assumiu 27 valores, que são os nomes das 12 instituições estrangeiras e das 25 instituições brasileiras às quais se vinculam ao menos um dos autores dos textos em análise.

Assim como no caso da classificação de fontes, classificação de código permite a filtragem pelos atributos de códigos e é fácil aplicar os atributos sobre os códigos livres também. Basta acessar a pasta dos códigos livres e, no canto direito da tela, realçado na Figura 4 com a seta vermelha, atribuir os valores dos atributos de classificação criados anteriormente. Vale lembrar que é importante gravar a operação pressionando o botão com o símbolo do disquete.

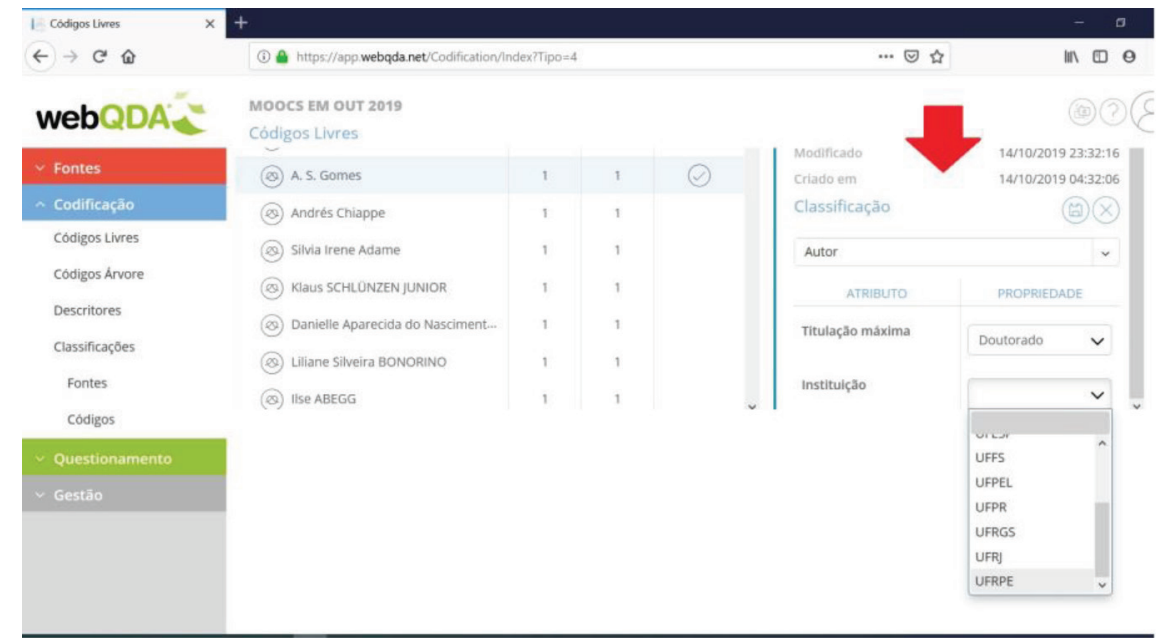

Figura 4 - Atribuir classificação de código na interface do software webQDA

Para codificar nas fontes as palavras-chave, os principais resultados e as lacunas reveladas pelos estudos, bem como para destacar os objetivos do trabalho, optou-se por realçar as passagens do texto por meio de "descritores". Os descritores funcionam como atributos de classificação a serem aplicados sobre um trecho da fonte em análise (Souza 
et al., 2016, p.22). Os descritores apresentados na Figura 5 foram aplicados aos trechos relativos a essas categorias.

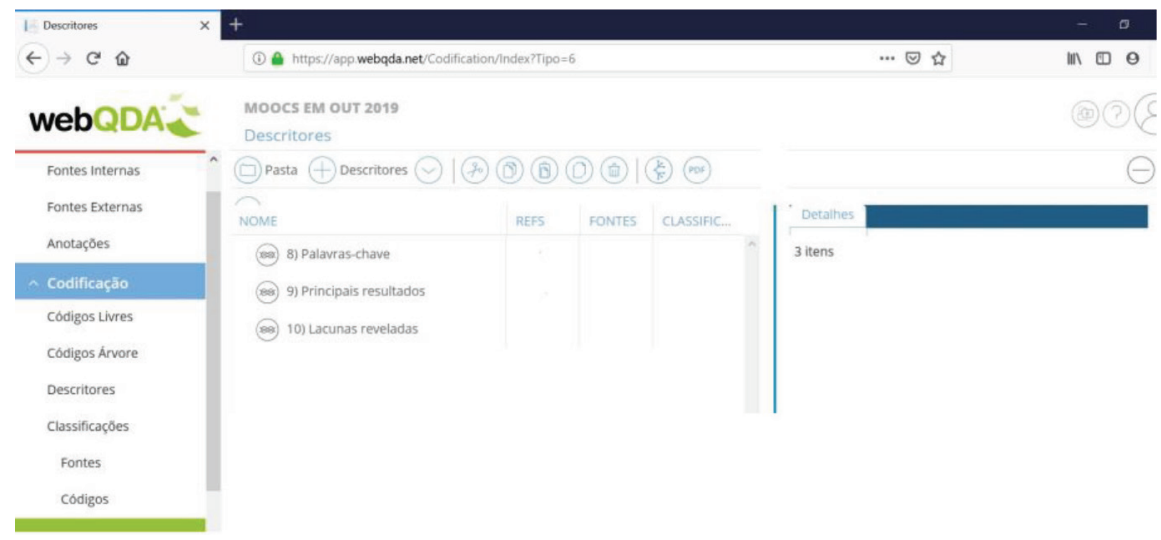

Figura 5 - Definir descritores de fonte na interface do software webQDA

Já para as categorias mais interpretativas, pautando os objetivos, o tema ou assunto principal abordado pelos artigos, optou-se por destacar as passagens de textos por meio de "códigos árvore", gerando uma estrutura hierárquica para organizar códigos e subcódigos, como se vê na Figura 6.

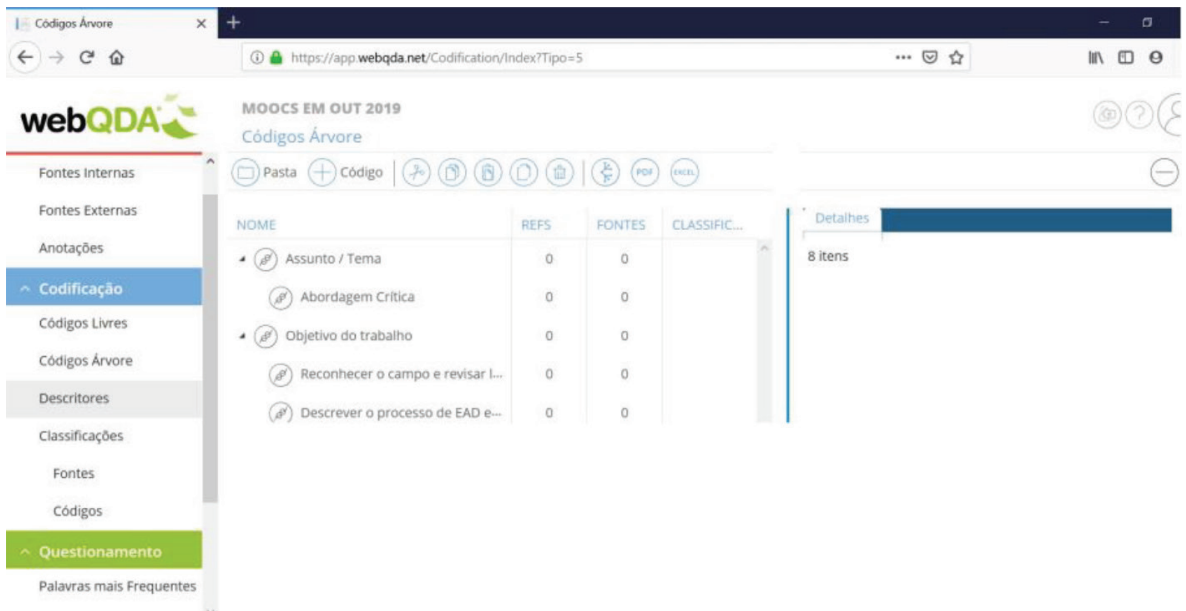

Figura 6 - Estrutura de códigos-árvore usada no estudo

A estrutura de classificação oferecida pelo software webQDA - composta por códigos, classificações por atributos e descritores, discutidos nos parágrafos acima, auxiliaram as análises apresentadas na seção seguinte. 


\section{Análises e discussões}

Depois de tratados os dados com o auxílio do software webQDA, foram geradas matrizes com cruzamentos das codificações e dessas com seus atributos. "As análises revelam que as revistas da área da Educação foram as campeãs em publicações sobre o tema (com 22 dos 29 artigos analisados), seguida pelas revistas da Administração (5 artigos)" (do Carmo et al., 2019, p. 586). A baixa produção acadêmica sobre MOOCs em outras áreas de conhecimento - que não seja a área de Educação - contrasta com a realidade de difusão dessa tecnologia de ensino em diferentes áreas científicas. A Revista Novas Tecnologias na Educação (Renote) foi aquela que concedeu maior espaço ao tema, tendo publicado seis artigos sobre MOOCs nos últimos 10 anos.

No que concerne à autoria da produção nacional sobre MOOCs, 89 autores estiveram envolvidos na produção analisada, dos quais $49 \%$ são doutores, $12 \%$ são doutorandos e 16\% são mestres. Apenas dois desses autores contam dois ou mais artigos no corpus de pesquisa, o que parece revelar que os MOOCs não estão dentre seus temas de investigação prioritários e que as publicações parecem derivar de iniciativas isoladas e pontuais dos autores.

A distribuição geográfica dos autores brasileiros envolvidos com produção sobre MOOCs revelaram concentração no sul/sudeste do país. A Universidade Federal do Rio Grande do Sul (UFRGS) e Universidade Federal de Santa Maria são as instituições de origem dos autores que concentram maior número de publicações, com três artigos cada uma. A Universidade de São Paulo (USP) e a Universidade Federal de Santa Catarina (UFSC) contam dois artigos cada.

Em onze trabalhos observa-se a presença de autores de mais de uma instituição, podendo apontar parceiras interinstitucionais. No Diretório de Grupos de Pesquisa no Brasil na Plataforma Lattes do Conselho Nacional de Desenvolvimento Científico e Tecnológico (CNPq), foram encontrados três grupos de pesquisa vinculados aos estudos de "MOOCs". No entanto, quanto a um desses grupos não foi encontrado no corpus de pesquisa nenhum trabalho de autoria de seus membros. No caso dos outros dois grupos de pesquisa foram analisados trabalhos publicados pelos pesquisadores a eles vinculados. Vale notar que alguns desses autores se vinculam a ambos os grupos, concomitantemente.

Os trabalhos teórico-empírico, de abordagem qualitativa e com recorte transversal predominam no corpus analisado. Os métodos de investigação predominantes foram os estudos de caso, os métodos mistos e a pesquisa documental, com coletas da dados online. A maioria dos trabalhos teórico-empírico aplicam a análises de conteúdo (50\%), sendo o segundo métodos analítico mais recorrente as análises estatísticas, sejam elas descritivas (18,18\%), inferenciais (4,55\%) ou ambas (9,09\%). Apenas dois apresentaram delineamento correlacional. Não foram encontrados trabalhos experimentais ou quase-experimentais.

\subsection{Evolução da Temática da Produção Nacional Sobre MOOCS}

Foi em 2013 que se publicou o primeiro trabalho sobre MOOCs nos periódicos brasileiros considerados nesta pesquisa $\mathrm{A}$ análise dos assuntos e dos objetivos das 
publicações analisadas revelou padrão evolutivo da abordagem da temática na produção brasileira, destacando-se duas fases: na primeira, de 2013 a 2015, os trabalhos buscaram reconhecer o campo e revisar a literatura. Já na segunda fase, que vai de 2016 a 2018, os trabalhos apresentaram experiências de aplicação dos MOOCs e aprofundamento dos estudos sobre a temática. A linha do tempo apresentada na Figura 7 sintetiza a evolução da produção científica brasileira sobre MOOCs.

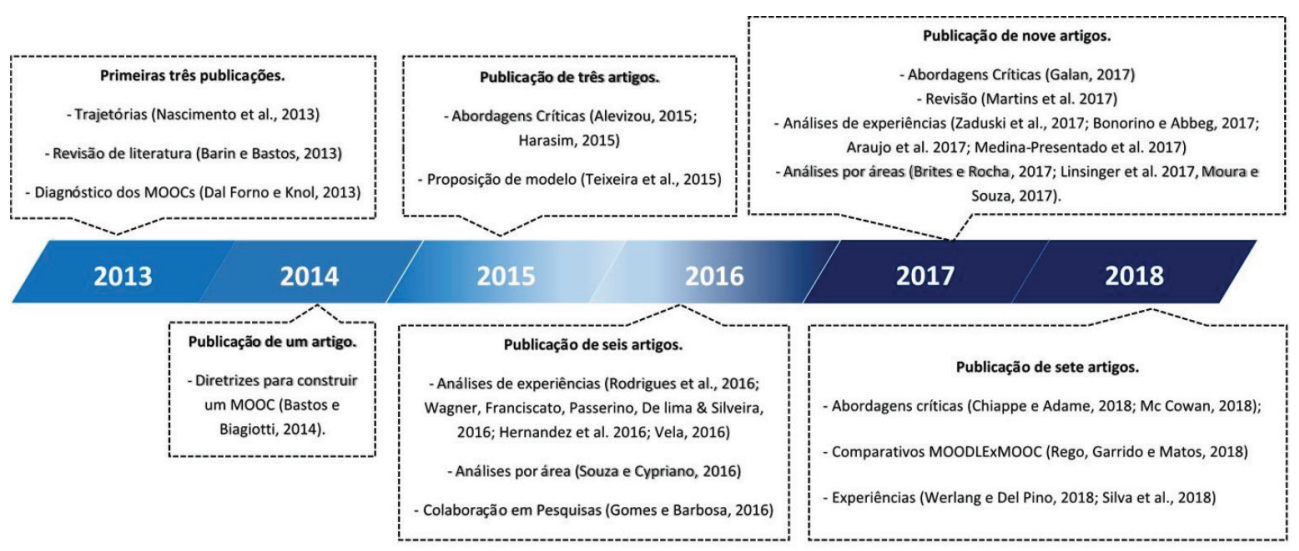

Figura 7 - Linha do tempo e evolução dos artigos sobe MOOcs publicados no Brasil

Na primeira fase, os trabalhos são teóricos, descritivos e incipientes, sem discussão ou propostas de trabalhos empíricos, constando revisões de literatura (Dal Forno, Barin \& Bastos, 2013; Knoll, 2013), descrição do processo de EAD que culmina no surgimento dos MOOCs (Nascimento et al., 2013) e sugestões para o incremento da eficiência de MOOCs (Cid Bastos \& Biagiotti, 2014). Vale realçar que até 2014, ainda se estavam implantando as primeiras experiências com MOOCs no contexto brasileiro.

Ao final dessa primeira fase, já em 2015, as publicações sobre MOOCs passam a se mostrar mais robustas. Três artigos internacionais foram publicados em revistas brasileiras., sendo que dois deles trabalhos trazem abordagens críticas aos MOOCs (Alevizou, 2015; Harasim, 2015) e um deles apresenta o modelo de MOOCs implementado na Universidade Aberta Portuguesa (UAb) (Teixeira et al., 2015).

No período de 2016 e 2018, ou seja, na segunda fase evolutiva da produção científica brasileira sobre MOOCs, a quantidade de publicações aumentou expressivamente (mais que triplo da produção da fase anterior). Juntamente com análises críticas, emergem estudos descritivos voltados para avaliação de experiências, desvelando a tendência de consolidação do MOOCs no cenário brasileiro. Nessa segunda fase, foram avaliadas experiências do uso de MOOCs no que concerne à aspectos funcionais das plataformas, perfis comportamentais dos usuários, percepção de eficiência e satisfação, percepção ou avaliação de aprendizagem.

Numa avaliação quanto à efetividade dos MOOCs, a partir de uma perspectiva ampla, Moura e Souza (2017) concluíram que eles apresentam baixo o potencial disruptivo, ou 
seja, não transformam os modelos educacionais de modo promover resultados de ensinoaprendizagem significativamente diferentes dos modelos educacionais tradicionais. Também aportando críticas aos MOOCs, Martins, Leite e Ramos (20017) alertam para o risco de que, ao mesmo tempo em contribuem para a internacionalização da educação, os MOOCs também podem se prestar uma nova forma de colonização legalizada.

\section{Considerações Finais}

O estudo de Gomes \& Barbosa (2016) revelou que a produção científica internacional sobre MOOCs intensificou-se nitidamente nos últimos cinco anos, o que converge com os resultados das análises deste trabalho. Este trabalho buscou oferecer uma visão panorâmica da produção científica sobre MOOCs publicada no Brasil. Segundo o panorama apresentado, eis um tema ainda pouco explorado pela produção científica. As análises, revelaram que grande parte dos trabalhos analisados são de caráter exploratório, e que a poucas experiências descritas no corpus de pesquisa revelam o pequeno potencial de inovação dos cursos implementados, especialmente com relação ao idioma e os formatos dos cursos.

A tendência crescimento e ampla difusão dos cursos em plataformas MOOC, no cenário brasileiro e internacional clama por se conhecer mais profundamente os princípios, os processos e as práticas dessa modalidade de educação a distância mediada por computador. Diante disso, se demandam investigações que revelem o perfil dos estudantes, os padrões de interação entre os participantes, suas estratégias de aprendizagens bem como a efetividade e a capacidade inovativa dos MOOCs.

Diante do alerta de Martins, Leite e Ramos (2017) e de Rambe e Moeti (2017), a produção científica não pode se furtar de também oferecer trabalhos críticos, que pautem a contribuição social e o potencial de democratização trazido pelos MOOCs, e que também indaguem sobre as motivações e sobre os protagonistas das ofertas desses cursos.

Observa-se tendência de crescimento da produção científica sobre os MOOCs, mas os dados revelam que as os autores dos trabalhos analisados não são pesquisadores assíduos da temática e que há poucos os grupos de pesquisas ativos e produtivos sobre o tema, de modo que se constata carência de consolidação como tema de investigação científica.

\section{Referências}

Alevizou, G. (2015). From OER to MOOCs: Critical perspectives on the historical mediation trajectories of open education. International Journal of Media \& Cultural Politics, 13(34), 347-378.

Araujo, R. T. S., Medeiros, F. N. S., Araujo, M. E. S., Lima, K. P., Araujo, N. M. S., \& Rodrigues, F. A. A. (2017). A statistical analysis of the learning effectiveness in online engineering courses. IEEE Latin America Transactions, 15(2), 300-309. DOI: https://doi.org/10.1109/TLA.2017.7854626

Barin, C. S., \& Bastos, F. P. (2013). Problematização dos MOOC na atualidade: potencialidades e Desafios. Novas Tecnologias na Educação, 11(3), 1-10. 
Brites, L. S., Rocha, C. M. F. (2017). Massive Open Online Courses (MOOCS): perfil dos cursos no campo da Saúde. Novas Tecnologias Na Educação, 15(1), 106-126.

Cid Bastos, R., \& Biagiotti, B. (2014). MOOCs: uma alternativa para a democratização do ensino. Novas Tecnologias na Educação, 12(1), 1-9.

Cronin, P., Ryan, F., \& Coughlan, M. (2008). Undertaking a Literature Review. Doing Postgraduate Research, 17(1), 411-429. DOI: https://doi. org/10.1177/107808747000500401.

Dal Forno, J. P., \& Knoll, G. F. (2013). Os MOOCs no mundo: um levantamento de cursos online abertos massivos. Nuances: Estudos Sobre Educação, 24(3), 178-194.

do Carmo, E. A., Araújo, C. L., Murce, P., \& Abbad, G. S. (2019). Revisão sistemática de literatura assistida pelo software de análise qualitativa WebQDA: um estudo sobre a produção científica brasileira sobre Massive Open Online Courses (MOOCs) nos últimos 10 anos. In: A. P. Costa, S. Oliveira e Sá, P. Castro, \& D. N. de Souza (Eds.). Atas do $8^{\circ}$ Congresso Ibero-Americano em Investigação Qualitativa (Volume 1 - Investigação Qualitativa em Educação) (pp. 580-589). Porto: Ludomedia. Consultado em https://www.proceedings.ciaiq.org/index.php/CIAIQ2019/article/ view/2197.

Fassbinder, A., Delamaro, M. E., \& Barbosa, E. F. (2014). Construção e uso de moocs: uma revisão sistemática. In Simpósio Brasileiro de Informática na Educação-SBIE (Vol. 25, No. 1, p. 332).

Fontana, M. V. L., \& Leffa, V. J. (2018). MOOCs para o Ensino de Línguas: um estudo em call desde uma perspectiva conectivista. Alfa revista Linguíst, 62(1), 75-89.

Gomes, R. R., \& Barbosa, M. W. (2016). Colaborações em Pesquisas sobre Educação à Distância: uma Análise de Redes Sociais de Autores. Revista Novas Tecnologias na Educação, 14(1), 1-10.

Harasim, L. (2015). Educação online e as implicações da inteligência artificial. Revista da FAEEBA - Educação e Contemporaneidade, 24(44), 25-39.

Higashi, R. M., Schunn, C. D., \& Flot, J. B. (2017). Different underlying motivations and abilities predict student versus teacher persistence in an online course. Educational Technology Research and Development, 65(6), 1471-1493. DOI: https://doi. org/10.1007/s11423-017-9528-z.

Luís, C., Rocha, Á., \& Marcelino, M. (2017). Acessibilidade em Ambientes Virtuais de Aprendizagem. RISTI - Revista Ibérica de Sistemas e Tecnologias de Informação, (27), 54-65. doi: 10.17013/risti.25.54-65.

Ma, L., \& Lee, C. S. (2018). Understanding the Barriers to the Use of MOOCs in a Developing Country: An Innovation Resistance Perspective. Journal of Educational Computing Research, 57(3), 1-20. DOI: https://doi.org/10.1177/0735633118757732

Martins, T. B., Leite, M. da S., \& Ramos, M. P. A. M. (2017). Cursos Online Abertos e Massivo no Brasil no Contexto da Internacionalização da Educação Superior. Revista Internacional de Educação Superior, 3(3), 604-623. DOI: https://doi. org/10.22348/riesup.v3i2.7774. 
Mejías, C. B., Gema, B. de la F., \& Melo, F. A. (2013). El Protagonismo de la Informacióndocumentación em Cooperación al Desarrollo: los recursos educativos abiertos en los procesos de e-learning. Informação \& Sociedade, 23(1), 105-115.

Nascimento, A., Cardoso, L. D. S., \& Rocchietti, M. (2013). Educação a distância e cooperação estratégica entre as instituições metodistas de educação superior na América Latina. Educação \& Linguagem, 16(1), 19-37. DOI: https://doi. org/10.15603/2176-1043/EL.v16n1p19-37.

Noe, R., Clarke, A., \& Klein, H.J. (2014). Learning in the Twenty-First-Century Workplace. Annual Review of Organizational Psychology and Organizational Behavior, 1, 245275. DOI: https://doi.org/10.1146/annurev-orgpsych-031413-091321.

Oliveira, M. L. de. (2017) Desenvolvimento e avaliação de um MOOC interativo para ensino de biologia celular. Retrieved from http://repositorio.unicamp.br/handle/ REPOSIP/322345

Rambe, P., \& Moeti, M. (2017). Disrupting and democratising higher education provision or entrenching academic elitism: towards a model of MOOCs adoption at African universities. Educational Technology Research and Development, 65(3), 631-651. DOI: https://doi.org/10.1007/s11423-016-9500-3.

Rodrigues, R. L., Ramos, J. L. C., Silva, J. C. S., \& Gomes, A. S. (2016). Discovery Engagement Patterns MOOCs Through Cluster Analysis. IEEE Latin America Transactions, 14(8). Doi: 10.1109/TLA.2016.7785943.

Ruano, L. E., Congote, E. L., \& Torres, A. E. (2016). Comunicación e interacción por el uso de dispositivos tecnológicos y redes sociales virtuales en estudiantes universitarios. RISTI-Revista Ibérica de Sistemas e Tecnologias de Informação, (19), 15-31. doi: 10.17013/risti.19.15-31.

Souza, F., Costa, A., Moreira, A., Souza, D., \& Freitas, F. (2016). WebQDA: manual de utilização rápida. Aveiro: UA Editora.

Souza, R. de, \& Cypriano, E. F. (2016). MOOC: uma alternativa contemporânea para o ensino de astronomia. Ciência \& Educação, 22(1), 65-80. DOI: https://doi. org/10.1590/1516-731320160010005.

Teixeira, A., Mota, J., Morgado, L., \& Spilker, M. J. (2015). iMOOC: Um Modelo Pedagógico Institucional para Cursos Abertos Massivos Online (MOOCs). Educação, Formação \& Tecnologias, 8(1), 4-12.

Vela, L. A. O. (2016). Colaboración y TIC en América Latina: el caso Interconectados. Revista Linhas, 17(33), 82-110.

Zhang, Q., Peck, K. L., Hristova, A., Jablokow, K. W., Hoffman, V., Park, E., \& Bayeck, R. Y. (2016). Erratum to: Exploring the communication preferences of MOOC learners and the value of preference-based groups: Is grouping enough?. Educational Technology Research and Development, 64(6), 809-837. DOI: https://doi. org/10.1007/s11423-016-9448-3. 